\title{
Variable field of view full field transmission X-ray microscope
}

$\underline{\text { Jun Lim }}^{1, *}$, Sangsul Lee ${ }^{1}$, Jaeyeon Park ${ }^{1}$, Yulan Roh ${ }^{1}$ and Yesul Kim²

1. Pohang Light Source-II, ITCC, Pohang, South Korea.

2. Sungkyunkwan University, Soft matter physics lab., Suwon, South Korea

* Corresponding author, email_limjun@postech.ac.kr

Full field transmission X-ray microscopy (TXM) based on zone plate is promising method for various research fields including biology, material science, and so on. State-of-the-art TXM provides $\sim 10 \mathrm{~nm}$ spatial resolution images with a field of view to tens of microns. In parallel, radiography has made tremendous advances in instrumentations as well as spatial resolution. It provides images of large objects like a full-sized mice or lamb, but it is hard to obtain sub-micron resolution images. In particular, for industry applications, statistical values obtained from large volume of high resolution images are important. So far, no microscope has been introduced to satisfy these demands. In order to meet the requirements, we devised a modified TXM that deliver Zernike phase contrast images of $400 \mathrm{um}$ field of view with $100 \mathrm{~nm}$ resolution. We utilized off-axis illumination scheme so that there is no stitching and sample motion. Eventually, combining with computed tomography, 400 cubic microns sample can be rendered in 3D with $100 \mathrm{~nm}$ spatial resolution. In addition, like an electron microscope with variable FOV, if we can make TXM with variable FOV it will open new scientific fields. In this study, we present a newly developed TXM setup that can adjust FOV from 50 um to $400 \mathrm{um}$.

Generally, TXM is consists of a capillary condenser, zone plate, and detector. All components are aligned on the optical axis. In terms of resolution, high resolution can be realized if the condenser and zone plate meet the NA matching condition. As for the field of view, it is limited by the beam size focused by the capillary condenser. Typically, it is around $50 \mathrm{um}$. Therefore, if the sample is larger than the fov, we have to stitch several images. However, stitching only works in 2D not 3D, because inevitably we have to move the sample vertically and horizontally. One way around the obstacle is to use a technique called oblique illumination microscope. If the illumination is directed so that it originates from a single azimuth and strikes the specimen from an oblique angle, details in the specimen may be revealed with a good contrast and visual clarity. Physically, phase and refractive index gradients in the specimen deflect the light, so that only the zeroth order and one or two sidebands of diffracted light can recombine at the image plane. This produces a relief-like specimen pattern having regions displaying shadows and highlights, much like that observed with the differential interference contrast (DIC) microscope. In order to extend FOV, we adopted the method of oblique illumination microscope. The concept is very simple. When collimated $\mathrm{x}$ rays incident to half of the zoneplate, the magnified sample image is formed on the opposite side of the detector. Another half image can be obtained in the same way. Although we have to stitch two images, it need not to move the sample at all. So we can get CT images by rotating sample in the usual way. The experiment was performed in BL7C spectroscopic X-ray Nano Imaging (sXNI) beamline at PLS-II. The beamline is designed for spectral nano-resolution-imaging utilizing zone-plate-based transmission $\mathrm{X}$-ray microscopy (TXM) [1,2]. Monochromatic X-rays are selected by a liquid-nitrogen-cooled double-crystal monochromator (DCM) and focused using a rhodium-coated horizontal focusing plane mirror (HFM) and a vertical focusing plane mirror (VFM). For microscopic imaging, an iridium objective zone plate of 30 $\mathrm{nm}$ outermost zone width, $400 \mu \mathrm{m}$ diameter and $0.6 \mu \mathrm{m}$ thickness is used. As a detector, a scintillatorcoupled optical microscope incorporating a thin scintillation crystal (15 $\mu$ m-thick Tb:LSO), a 20X optical objective and a large-area CCD with $4096 \times 4096$ sensors of $9 \mu \mathrm{m}$ pixel size is utilized. As for an example, 
we imaged a part of Intel Pentium-4 processor (Fig. 1(3)). In large fov mode, 400 um fov with $100 \mathrm{~nm}$ pixel resolution achieved (Fig. 1(1)). It shows only large scale nested microwires, but it helps to find out which parts zoom in on. In zoom in mode, we can see micron and sub-micron size pillar structures connecting each layer, which provides $50 \mathrm{um}$ fov with $30 \mathrm{~nm}$ resolution image (Fig. 1(2)). Based on these results, we confirmed the possibility, and plan to get CT images of the microchip in the zoom out and zoon in mode in the next step.

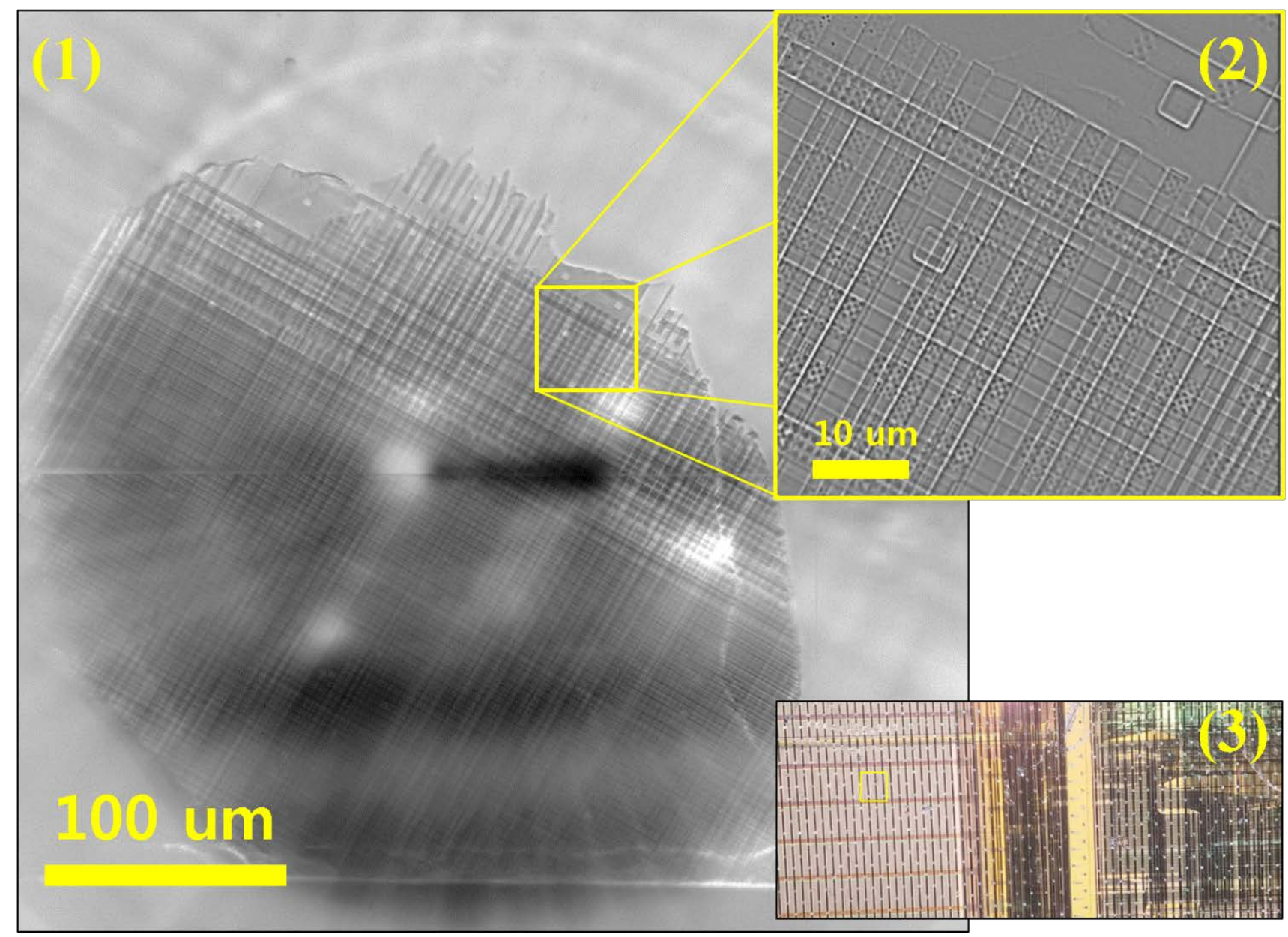

Figure 1. (1) Zoom out and (2) zoom in view of (3) a part of Intel Pentium-4 microprocessor.

[1] Sangsul Lee et al, J. Synchrotron Rad. 24 (2017) 1276

[2] Jun Lim et al, J. Synchrotron Rad. 21 (2014) 827 